\title{
MODELOS DE VOLUME E AFILAMENTO PARA FLORESTAS DE Pinus taeda $\mathrm{L}$.
}

\author{
VOLUME AND TAPER MODELS FOR Pinus taeda L. FORESTS \\ Ricardo Murilo Malheiros dos Santos ${ }^{1}$, Andrea Nogueira Dias², Julio Eduardo Arce ${ }^{3}$, Vlademir \\ Martarello ${ }^{4}$, Edson Luis Serpe ${ }^{5}$, Thiago Floriani Stepka ${ }^{6}$, Gerson dos Santos Lisboa ${ }^{7}$ \\ 1,2,5 Universidade Estadual do Centro-Oeste, Irati, Paraná, Brasil-ricardomurilo@msn.com, \\ anogueiradias@hotmail.com \& serpe.edson@gmail.com \\ ${ }^{3}$ Universidade Federal do Paraná, Curitiba, Paraná, Brasil-jarce@ufpr.br \\ ${ }^{4}$ ADAMI, Caçador, Santa Catarina, Brasil-vlademir.martarello@adami.com.br \\ ${ }^{6}$ Universidade do Estado de Santa Catarina, Lages, Santa Catarina, Brasil - tfstepka@yahoo.com.br \\ ${ }^{7}$ Universidade Federal do Sul da Bahia, Itabuna, Bahia, Brasil-gerson.lisboa@gmail.com
}

\section{RESUMO}

\begin{abstract}
A gama de produtos e recursos florestais existentes no mercado exigem metodologias precisas na quantificação do estoque de um povoamento. Este trabalho teve como objetivo a modelagem de volume e afilamento para florestas de Pinus taeda L. na região oeste do Estado de Santa Catarina. Foram ajustados modelos de volume e afilamento considerando: (1) para o conjunto de dados de árvores cubadas, (2) estratificando os dados para os blocos A (Chapecozinho) e B (Cachoeira), e (3) dentro de cada bloco os dados foram estratificados por classes de idade. Os modelos foram ajustados como proposto por Schumacher-Hall e Spurr e a função de afilamento utilizada foi proposta por Schöepfer, ajustada em todas as situações analisadas. Dentre os modelos volumétricos, o de Schumacher-Hall mostrou-se superior ao modelo de Spurr, no ajuste do conjunto total de dados e para os blocos. Em relação ao modelo de afilamento ajustado, este se mostrou adequado e com estatísticas satisfatórias para determinar o volume por sortimento, com a finalidade de obter-se multiprodutos. Pela dispersão dos resíduos e análise do erro padrão de estimativa em porcentagem a estratificação dos dados em classes de idade demonstrou-se superior.
\end{abstract}

PALAVRAS-CHAVE: Inventário florestal, Planejamento, Sortimento.

\section{ABSTRACT}

The range of products and existing forest resources in the market require accurate methods in quantification of a stand. The objective of this work was the modeling of volume and tapering for forests of Pinus taeda $\mathrm{L}$. in the western region of the State of Santa Catarina. The volume models and taper functions were adjusted considering: (1) for the cubed tree data set, (2) stratifying the data by Chapecozinho block (A) and Cachoeira blocks (B), and (3) within each Block the data were stratified by age classes. The models were adjusted as proposed by Schumacher-Hall and Spurr and the grinding function used was proposed by Schöepfer, adjusted in all situations analyzed. Among the volumetric models, the Schumacher-Hall model was superior to the Spurr model, in the fit of the total data set and for the blocks. In relation to the adjusted model of tapering, this was adequate and with satisfactory statistics to determine the volume by assortment, in order to obtain multiproducts. By dispersion of the residuals and analysis of the standard error of estimate in percentage, the stratification of the data in age classes was shown superior.

KEY WORDS: Forest inventory, Planning, Assortment. 


\section{INTRODUÇÃO}

A Indústria Brasileira de Árvores (IBÁ, 2017) apontou os estados do Paraná e Santa Catarina como os maiores produtores de madeira de Pinus spp. do Brasil. A região conta com diversas empresas do ramo florestal que comercializam e consomem produtos para laminação, serraria, celulose e diversas outras finalidades.

O uso de equações volumétricas é uma das principais ferramentas para quantificação da produção de povoamentos florestais (LEITE \& ANDRADE, 2003).

As funções de afilamento são ferramentas para determinação do volume de árvores e toras por permitirem estimar três características básicas das árvores: diâmetros em qualquer ponto do fuste, altura no fuste em que se encontra um determinado diâmetro e o volume entre dois pontos quaisquer no fuste (PRODAN et al., 1997).

Scolforo (1993) comenta que, em termos de precisão, as funções de afilamento e as equações de volume são equivalentes, porém, a primeira é muito mais interessante na medida em que consegue estimar o volume de qualquer porção de árvore.

A amostragem para geração de equações de volume para florestas equiâneas é feita usualmente, por espécie, espaçamento, classe de idade e regime de corte (CAMPOS \& LEITE, 2013) e, neste caso, estimam bem o estoque e volume individual de árvores. No caso das funções de afilamento, sua utilização é estritamente importante para determinar o sortimento de cada indivíduo, a partir da integral da função.

Figueiredo Filho et al. (2014), em um compêndio de equações de volume e afilamento para espécies exóticas e nativas das regiões do Brasil, destacam a importância destas ferramentas para os profissionais da área florestal, para avaliar e determinar estoque e sortimento da produção atual e futura de florestas.

Oliveira et al. (2009) relatam que o modelo de Schumacher-Hall (1933) é o mais utilizado por empresas do setor privado, enquanto que o modelo de Schöepfer (1966), conhecido também como "polinômio de quinto grau", é uma função de afilamento muito utilizada pela sua versatilidade, cálculo da integral, além de gerar boas estimativas.

Com vista a maximização de receitas ou produção, toda melhora em estimativas que possam ser efetuadas, tem grande relevância. Autores como Souza (2009), Kohler et al. (2013) estudando Pinus taeda L., Machado et al. (2004) estudando Pinus oocarpa Schiede, revelam ganhos em exatidão estratificando os dados de alguma forma, seja por classe de diâmetro, idade, regimes de manejo ou forma do tronco.

Esta pesquisa tem como objetivos, ajustar modelos de volume e afilamento para um conjunto total de dados; para os dados estratificados por regiões (denominados blocos); e para os dados de blocos estratificados por classe de idade.

\section{MATERIAL E MÉTODOS}

Os dados são oriundos de cubagem provenientes de florestas de $P$. taeda que abrangem a região Oeste de Santa Catarina, Brasil. Essas florestas pertencem a uma empresa que conta com dois grandes maciços florestais, inseridos em regiões distantes a mais de $90 \mathrm{~km}$, de forma que dois blocos podem ser observados. A divisão dos blocos florestais pode ser observada na Figura 1.

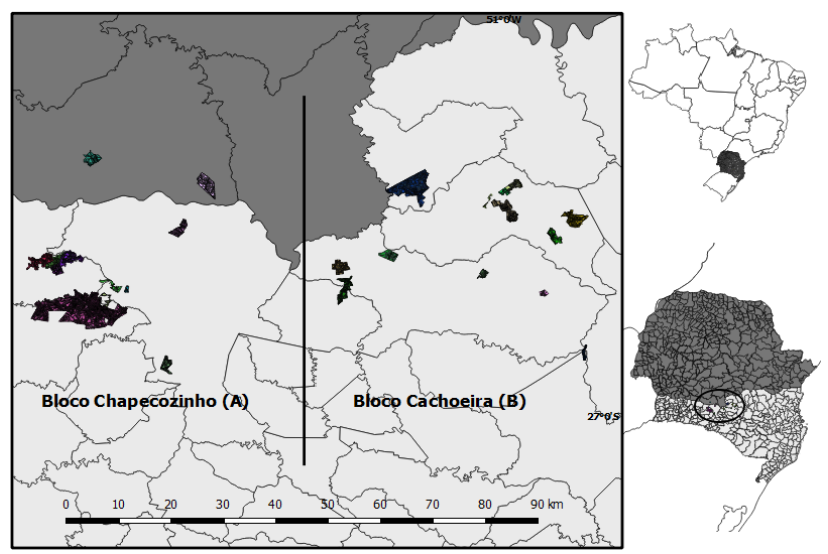

Figura 1. Bloco A (Chapecozinho); bloco B (Cachoeira).

O conjunto total de árvores cubadas, considerando os dois blocos, foi de 1.314 árvores, sendo 612 árvores cubadas no bloco A e 702 árvores cubadas no bloco B.

A cubagem foi realizada pelo método de Smalian, utilizando seções relativas (método de Hohenadl) com 15 seções, que correspondem às alturas relativas: 0,$5 ; 1 ; 5 ; 10$; $15 ; 20 ; 25 ; 30 ; 40 ; 50 ; 60 ; 70 ; 80 ; 90$ e $95 \%$ da altura total. Para a modelagem ainda foi utilizado a altura a $100 \%$ e di (diâmetro tomado a i-ésima altura) correspondente igual a 0 .

Para os ajustes, os dados foram estratificados conforme a definição do manejo, 1어 desbaste (entre 7 e 10 anos), 2은 desbaste (entre 11 e 14 anos), 3ㅇ desbaste (entre 15 e 18 anos) e corte raso (entre 19 e 23 anos) (Figura 2). 


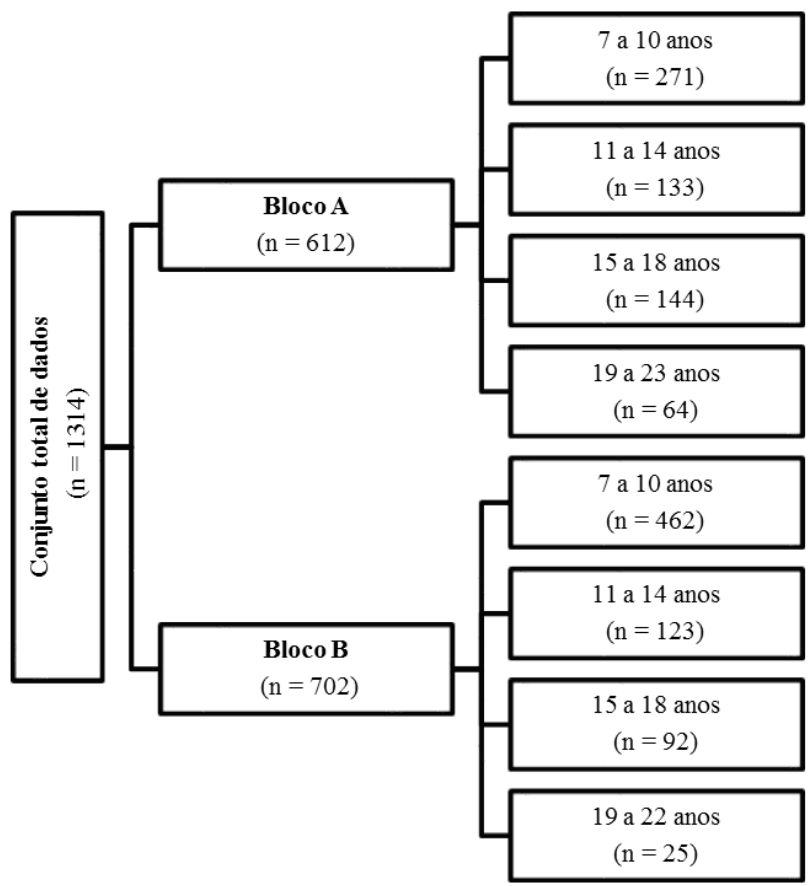

Figura 2. Estratificação dos dados de cubagem para a modelagem de volume e afilamento.

Foram testados modelos de volume e afilamento, conforme descritos nas Tabelas 1 e 2, os quais foram escolhidos por serem os mais utilizados pelas empresas da região da área de estudo. Os modelos foram ajustados considerando todos os dados, sendo utilizando planilhas eletrônicas e o software Florexcel (OPTIMBER, 2016), na sequência os modelos foram ajustados para cada bloco e então para cada classe de idade, dentro de cada bloco.

Tabela 1. Modelos de volume.

\begin{tabular}{cc}
\hline \multicolumn{1}{c}{ Modelos } & Autor \\
\hline $\mathrm{v}=\mathrm{b}_{0}+\mathrm{b}_{1} \cdot \mathrm{d}^{2} \mathrm{~h}$ & Spurr (1952) \\
$\operatorname{lnv}=\mathrm{b}_{0}+\mathrm{b}_{1} \cdot \operatorname{lnd}+\mathrm{b}_{2} \cdot \operatorname{lnh}$ & Schumacher-Hall $(1933)$ \\
\hline $\mathrm{v}=$ volume individual $\left(\mathrm{m}^{3}\right) ; \mathrm{d}=$ diâmetro medido na altura de $1,3 \mathrm{~m}(\mathrm{~cm}) ;$ \\
$\mathrm{e} h=$ altura total $(\mathrm{m})$.
\end{tabular}

Os modelos de volume ajustados para o conjunto de dados e blocos foram Schumacher-Hall e Spurr, para as classes de idade, utilizou-se o modelo de Schumacher-Hall. A função de afilamento (Schöepfer - Polinômio de quinto grau), foi ajustada em todas as situações analisadas.

Tabela 2. Função de afilamento.

\begin{tabular}{cc}
\hline Modelos & Autor \\
\hline$\frac{\mathrm{di}}{\mathrm{DAP}}=\mathrm{b}_{0}+\mathrm{b}_{1} \frac{\mathrm{hi}}{\mathrm{h}}+\mathrm{b}_{2}\left(\frac{\mathrm{hi}}{\mathrm{h}}\right)^{2} \ldots \mathrm{b}_{5}\left(\frac{\mathrm{hi}}{\mathrm{h}}\right)^{5}$ & Schöepfer (1966) \\
\hline
\end{tabular}

$\overline{\mathrm{di}}=$ diâmetro na altura $h i(\mathrm{~cm}) ;$ dap = diâmetro medido na altura de 1,3 $\mathrm{m}$ $(\mathrm{cm}) ; \mathrm{hi}=$ distância do solo até o ponto onde o diâmetro di é considerado $(\mathrm{m}) ;$ e $\mathrm{h}=$ altura total $(\mathrm{m})$.
Para eliminar a discrepância logarítmica do modelo de Schumacher, os valores estimados pela equação logaritmizada foram corrigidos multiplicando-se os volumes estimados $\left(\mathrm{m}^{3} . \mathrm{ha}^{-1}\right)$ pela expressão conhecida como Índice de Meyer (IM):

$$
\mathrm{IM}=\mathrm{e}^{0,5 \cdot s_{y x}^{2}}
$$

Em que: IM= Índice de Meyer; e = base dos logaritmos neperianos; e $\mathrm{S}_{\mathrm{yx}}{ }^{2}$ = quadrado do erro-padrão da estimativa em unidades logarítmicas.

Para a seleção do melhor modelo foram analisadas as seguintes estatísticas: coeficiente de determinação ajustado $\left(R^{2}{ }^{2}\right.$ dj $)$, erro padrão de estimativa em metro cúbico para os modelos de volume e em centímetros para a função de afilamento e também em percentagem $\left(S_{y x} e\right.$ $\mathrm{S}_{\mathrm{yx}} \%$ ), além da análise gráfica dos resíduos. Os modelos logaritmizados tiveram seus erros e coeficientes de determinação recalculados para a variável de interesse.

\section{RESULTADOS E DISCUSSÃO}

Ajuste dos modelos considerando o conjunto total de dados

Os coeficientes e as estatísticas considerando a cubagem das 1314 árvores de P. taeda, ou seja, todo o conjunto de dados está apresentado na Tabelas 3 e 4.

Tabela 3. Coeficientes e estatísticas para os modelos de volume.

\begin{tabular}{|c|c|c|c|c|c|}
\hline Modelos & \multicolumn{2}{|c|}{ Coeficientes } & $\mathbf{R}_{\text {adj }}$ & $S_{y x}\left(m^{3}\right)$ & $S_{x y}(\%)$ \\
\hline \multirow{3}{*}{ Schumacher-Hall } & $b_{0}$ & $-10,049159$ & \multirow{3}{*}{0,986} & \multirow{3}{*}{0,0416} & \multirow{3}{*}{11,80} \\
\hline & $b_{1}$ & 1,966127 & & & \\
\hline & $b_{2}$ & 0,977161 & & & \\
\hline \multirow{2}{*}{ Spurr } & $b_{0}$ & 0,008742 & \multirow{2}{*}{0,985} & \multirow{2}{*}{0,0485} & \multirow{2}{*}{13,87} \\
\hline & $b_{1}$ & $3,5433 \mathrm{E}-05$ & & & \\
\hline
\end{tabular}

$\mathrm{O}$ ajuste dos modelos de volume de Schumacher-Hall e de Spurr resultaram em valores de $\mathrm{R}^{2}$ adj semelhantes: 0,986 e 0,985 respectivamente. Assim como o coeficiente de determinação ajustado o erro padrão da estimativa também apresentou valores muito próximos entre os modelos ajustados: 0,0416 e 0,0485 $\mathrm{m}^{3}$. O erro em porcentagem foi maior para o modelo de Spurr, com $13,87 \%$, enquanto o modelo de Shumacher-Hall apresentou $11,80 \%$. 
Tabela 4. Coeficientes e estatísticas para a função de afilamento.

\begin{tabular}{lccccc}
\hline Modelos & Coeficientes & $\mathbf{R}_{\text {adj }}$ & $\mathbf{S}_{\mathbf{y x}}(\mathbf{c m})$ & $\mathbf{S}_{\mathbf{x y}}(\%)$ \\
\hline & $b_{0}$ & 1,243829 & & & \\
& $b_{1}$ & $-3,888341$ & & & \\
Schöepfer & $b_{2}$ & 16,107444 & & & \\
& $b_{3}$ & $-34,951103$ & & & \\
& $b_{4}$ & 33,086277 & & & \\
& $b_{5}$ & $-11,597225$ & & & \\
\hline
\end{tabular}

Em geral, os dois modelos de volume analisados conferem adequada estimativa da variável dependente e apresentam estatísticas semelhantes.

Kohler et al. (2012), encontraram resultados semelhantes ao deste estudo $\left(R^{2}\right.$ variando de 0,98 até 0,99 e $S_{y x}(\%)$ variando de $8,99 \%$ até $\left.14,37 \%\right)$, independentemente do método de ajuste do modelo de Schumacher-Hall, analisando a forma linear e não linear do modelo de Schumacher-Hall sob diferentes métodos (Marquardt, Steepest descent, Gauss-Newton e também pelo Solver do Excel).

Yoshitani Junior (2009), trabalhando com equações de volume para $P$. taeda da região Centro-Sul de Santa Catarina, também encontrou os resultados superiores para os modelos de Schumacher-Hall e Spurr, embora este último modelo tenha sido ajustado na forma linearizada. 0 autor ainda relacionou as estimativas de modelos de volume de simples e dupla entrada, DAP e DAP e altura respectivamente, e enfatiza que os modelos de dupla entrada apresentam estatísticas superiores em relação aos modelos de simples entrada.

A função de afilamento do polinômio de quinto grau apresentou um ajuste com 0,988 de $\mathrm{R}^{2}$ adj e um erro de estimativa em porcentagem de $7,28 \%$.

Os gráficos de distribuição dos resíduos dos modelos de volume (Figura 3), mostra que o modelo de Spurr teve uma tendência maior em superestimar os volumes nas menores classes de DAP em relação ao modelo de Schumacher-Hall.
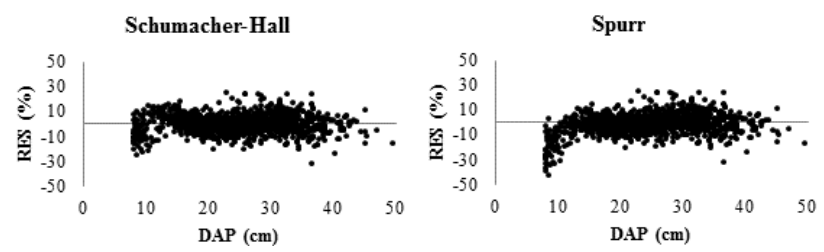

Figura 3. Distribuição de resíduos dos modelos de volume.

Os resíduos da função de afilamento do polinômio de quinto grau (Figura 4) ajustado a todo o conjunto de dados mostrou tendência para as últimas medidas ajustadas, onde o erro de estimativa é maior, passando de $100 \%$ de diferença entre o ajustado e o estimado, tanto superestimando, quanto subestimando.

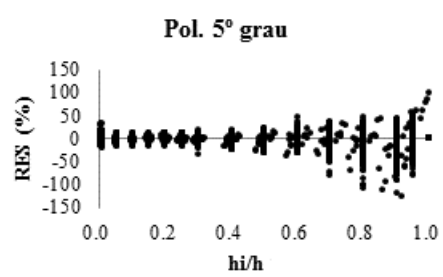

Figura 4. Distribuição de resíduos da função de afilamento.

\section{Ajuste dos modelos com estratificação em blocos}

Em relação aos blocos, ambos apresentaram estatísticas e coeficientes semelhantes entre si (Tabela 5), porém o bloco B (Cachoeira) apresentou os erros em porcentagem ligeiramente superiores ao bloco $A$ (Chapecozinho).

Tabela 5. Coeficientes e estatísticas considerando duas regiões, bloco $A$ (Chapecozinho) e bloco B (Cachoeira).

\begin{tabular}{|c|c|c|c|c|c|c|}
\hline & Modelos & & eficientes & $R^{2}$ adj & Sxy & Sxy (\%) \\
\hline & & $b_{0}$ & $-10,060730$ & & & \\
\hline & Schumacher & $b_{1}$ & 1,967855 & 0,984 & $\begin{array}{c}0,0558 \\
\left(\mathrm{~m}^{3}\right)\end{array}$ & 10,39 \\
\hline & & $b_{2}$ & 0,978816 & & & \\
\hline & & $b_{0}$ & 0,014366 & & 0,0564 & \\
\hline$\varangle$ & spurr & $b_{1}$ & 0,000035 & 0,984 & $\left(\mathrm{~m}^{3}\right)$ & 10,51 \\
\hline \&̊ํํ & & $b_{0}$ & 1,234144 & & & \\
\hline & & $b_{1}$ & $-3,836638$ & & & \\
\hline & Schöenfer & $b_{2}$ & 15,896376 & 0989 & 1,2271 & 711 \\
\hline & 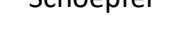 & $b_{3}$ & $-34,325822$ & 7, & $(\mathrm{cm})$ & 8,11 \\
\hline & & $b_{4}$ & 32,309952 & & & \\
\hline & & $b_{5}$ & $-11,277664$ & & & \\
\hline & & $b_{0}$ & $-10,060730$ & & & \\
\hline & Schumacher & $b_{1}$ & 1,967855 & 0,985 & $\begin{array}{c}0,0408 \\
\left(m^{3}\right)\end{array}$ & 11,57 \\
\hline & & $b_{2}$ & 0,978816 & & & \\
\hline & urr & $b_{0}$ & 0,014366 & 985 & 0,0399 & 1131 \\
\hline & מרוד & $b_{1}$ & 0,000035 & נינ, & $\left(m^{3}\right)$ & $11, \mathrm{~J} \perp$ \\
\hline 응 & & $b_{0}$ & 1,234144 & & & \\
\hline & & $b_{1}$ & $-3,836638$ & & & \\
\hline & Schöepfer & $b_{2}$ & 15,896376 & 0,987 & 1,1783 & 7,35 \\
\hline & & $b_{3}$ & $-34,325822$ & & $1 \mathrm{rm}$ & \\
\hline & & $b_{4}$ & 32,309952 & & & \\
\hline & & $b_{5}$ & $-11,277664$ & & & \\
\hline
\end{tabular}


Em relação ao conjunto total de dados, os ajustes mostram-se muito semelhantes, no ajuste por blocos foi encontrado para o bloco $\mathrm{A}, 0,0558 \mathrm{~m}^{3}$ para o erro padrão de estimativa $\left(S_{y x}\right)$ e $10,39 \%$ de erro em porcentagem ( $S_{y x} \%$ ) no modelo de Schumacher-Hall, enquanto que para modelo de Spurr apresentou $0,0564 \mathrm{~m}^{3}$ e 10,51\% para estas mesmas estatísticas.

Para o bloco $B$ os erros foram de $0,0408 \mathrm{~m}^{3}$ para o erro padrão de estimativa $\left(S_{y x}\right)$ e $11,57 \%$ do erro em porcentagem para o modelo de Schumacher-Hall. $O$ modelo de Spurr demonstrou valores de 0,0399 $\mathrm{m}^{3}$ e $11,31 \%$ de erros de estimativa e em porcentagem.

Quando se analisa o ajuste do polinômio de quinto grau para o conjunto total de dados e para cada bloco, observase ganho no ajuste para os dados do bloco $\mathrm{A}$ em relação ao erro padrão de estimativa em porcentagem $(7,11 \%$ contra $7,28 \%$ ) e ganho no ajuste do bloco $B$ em relação ao erro de padrão de estimativa $(1,1783 \mathrm{~cm}$ contra $1,2088 \mathrm{~cm})$.

Nas Figuras 5 e 6 pode-se observar a dispersão dos resíduos em porcentagem para os modelos de volume de Schumacher e Spurr e a função de afilamento para cada um dos blocos.
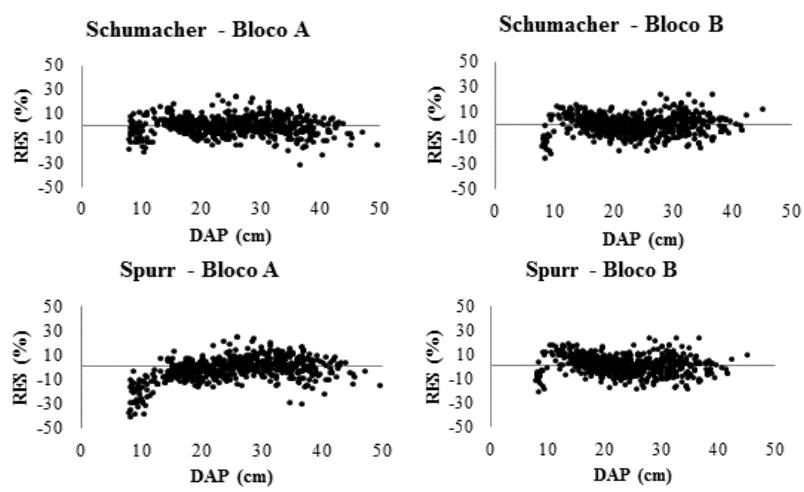

Figura 5. Dispersão dos resíduos para os modelos de volume no bloco A (Chapecozinho) e bloco B (Cachoeira).

Os ganhos da estratificação ficam evidentes na análise da dispersão de resíduos. Enquanto que os gráficos da dispersão de resíduos dos modelos volumétricos apresentam a dispersão em porcentagem da diferença do volume observado pelo estimado por classes de DAP, os gráficos da distribuição de resíduos da função de afilamento demonstram a diferença, em porcentagem, do di estimado com o di observado em classes de alturas relativas.

Quando comparados aos resíduos do conjunto total de dados, as dispersões dos resíduos dos blocos apresentam menores de discrepância das estimativas, tendo em vista a mesma escala do eixo $\mathrm{Y}$ dos resíduos.

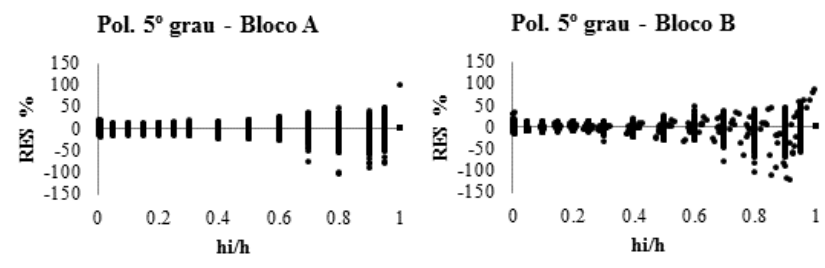

Figura 6. Dispersão dos resíduos para a função de afilamento no bloco A (Chapecozinho) e bloco B (Cachoeira).

\section{Ajuste dos modelos em classes de idade para o bloco $\mathrm{A}$}

Os dados foram também estratificados por classe de idade dentro de cada bloco. $\mathrm{Na}$ Tabela 6 estão apresentados os coeficientes e as estatísticas do modelo de volume de Schumacher-Hall e da função de afilamento do polinômio de quinto grau por classe de idade para o bloco A. Nas Figuras 6 e 7 pode ser observada a dispersão dos resíduos.

Tabela 6. Coeficientes e estatísticas por classe de idade para o bloco A (Chapecozinho).

\begin{tabular}{|c|c|c|c|c|c|c|c|}
\hline \multicolumn{2}{|c|}{$\begin{array}{c}\text { Classe de } \\
\text { idade }\end{array}$} & \multirow{2}{*}{$\begin{array}{c}\text { Modelos } \\
\text { Shumacher }\end{array}$} & \multicolumn{2}{|c|}{ Coeficientes } & \multirow{2}{*}{$\begin{array}{l}\mathbf{R}^{\mathbf{2}} \mathbf{a d j} \mathbf{j} \\
0,988\end{array}$} & \multirow{2}{*}{$\begin{array}{c}\mathbf{S}_{\mathbf{x y}} \\
\begin{array}{c}0,0124 \\
\left(\mathrm{~m}^{3}\right)\end{array}\end{array}$} & \multirow{2}{*}{$\begin{array}{r}\mathbf{S}_{\mathbf{x y}}(\%) \\
6,37\end{array}$} \\
\hline \multirow{8}{*}{ 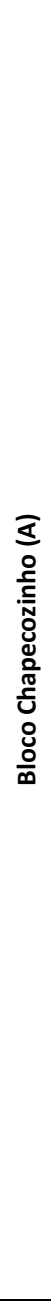 } & & & $\begin{array}{l}b_{0} \\
b_{1} \\
b_{2}\end{array}$ & $\begin{array}{c}-9,545625 \\
1,945106 \\
0,800145\end{array}$ & & & \\
\hline & $\begin{array}{c}7 \\
\text { a } \\
10 \\
\text { anos }\end{array}$ & Schöepfer & $\begin{array}{l}b_{0} \\
b_{1} \\
b_{2} \\
b_{3} \\
b_{4} \\
b_{5}\end{array}$ & $\begin{array}{c}1,266517 \\
-3,804103 \\
15,217568 \\
-33,619837 \\
32,722283 \\
-11,779169 \\
\end{array}$ & 0,990 & $\begin{array}{c}0,8157 \\
(\mathrm{~cm})\end{array}$ & 6,11 \\
\hline & & Shumacher & $\begin{array}{l}b_{0} \\
b_{1} \\
b_{2}\end{array}$ & $\begin{array}{c}-10,127068 \\
1,978204 \\
0,982191\end{array}$ & 0,981 & $\begin{array}{c}0,0431 \\
\left(\mathrm{~m}^{3}\right)\end{array}$ & 8,70 \\
\hline & $\begin{array}{c}11 \\
a \\
14 \\
\text { anos }\end{array}$ & Schöepfer & $\begin{array}{l}b_{0} \\
b_{1} \\
b_{2} \\
b_{3} \\
b_{4} \\
b_{5}\end{array}$ & $\begin{array}{c}1,218093 \\
-3,892796 \\
16,383004 \\
-35,290522 \\
33,048281 \\
-11,467775 \\
\end{array}$ & 0,992 & $\begin{array}{c}1,0231 \\
(\mathrm{~cm})\end{array}$ & 6,18 \\
\hline & & Shumacher & $\begin{array}{l}\text { b0 } \\
\text { b1 } \\
\text { b2 }\end{array}$ & $\begin{array}{c}-9,363984 \\
1,737735 \\
1,018378\end{array}$ & 0,950 & $\begin{array}{c}0,0630 \\
\left(\mathrm{~m}^{3}\right)\end{array}$ & 6,93 \\
\hline & $\begin{array}{c}15 \\
a \\
18 \\
\text { anos }\end{array}$ & Schöepfer & $\begin{array}{l}b_{0} \\
b_{1} \\
b_{2} \\
b_{3} \\
b_{4} \\
b_{5}\end{array}$ & $\begin{array}{c}1,202838 \\
-3,818645 \\
16,301312 \\
-34,319283 \\
31,072867 \\
-10,441393 \\
\end{array}$ & 0,987 & $\begin{array}{c}1,3898 \\
(\mathrm{~cm})\end{array}$ & 6,29 \\
\hline & & Shumacher & $\begin{array}{l}b_{0} \\
b_{1} \\
b_{2}\end{array}$ & $\begin{array}{c}-8,931904 \\
1,679256 \\
0,958254\end{array}$ & 0,920 & $\begin{array}{c}0,0939 \\
\left(\mathrm{~m}^{3}\right)\end{array}$ & 7,64 \\
\hline & $\begin{array}{c}19 \\
a \\
23 \\
\text { anos }\end{array}$ & Schöepfer & $\begin{array}{l}b_{0} \\
b_{1} \\
b_{2} \\
b_{3} \\
b_{4} \\
b_{5}\end{array}$ & $\begin{array}{c}1,201113 \\
-3,892679 \\
16,808996 \\
-35,254198 \\
31,765588 \\
-10,630495 \\
\end{array}$ & 0,986 & $\begin{array}{c}1,604 \\
(\mathrm{~cm})\end{array}$ & 6,60 \\
\hline
\end{tabular}



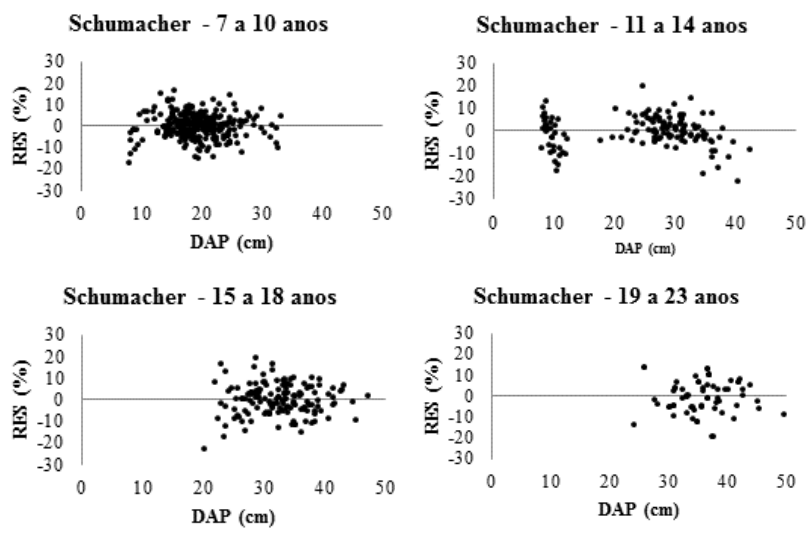

Figura 6. Dispersão dos resíduos do ajuste de volume para o bloco A (Chapecozinho).
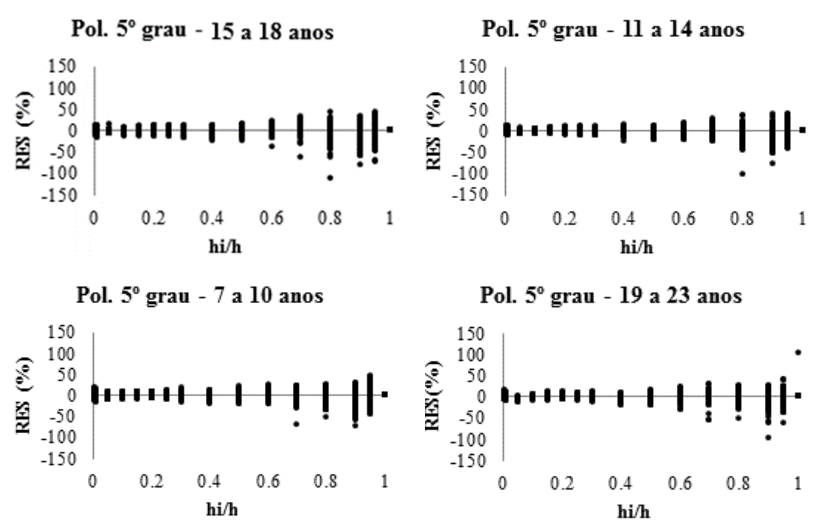

Figura 7. Dispersão dos resíduos do ajuste de afilamento para o bloco A (Chapecozinho).

\section{Ajuste dos modelos em classes de idade para o bloco B}

$\mathrm{Na}$ Tabela 7 observam-se os resultados encontrados para o bloco B considerando a estratificação dos dados em classes de idade. As estatísticas estão próximas as encontradas para o bloco A e denotam significativo decréscimo nos erros em porcentagem para cada classe de idade quando comparado ao conjunto total dados, e, quando observado o ajuste por bloco.

Nas Figuras 8 e 9 pode-se observar a dispersão de resíduos, que assim como no ajuste por classe de idade para o bloco $A$, houve ligeira melhoria em relação a tendenciosidade dos ajustes para o modelo de volume, enquanto que para o modelo de afilamento fica mais evidente que a dispersão dos resíduos diminuiu.
Tabela 7. Coeficientes e estatísticas por classe de idade para o bloco B (Cachoeira).

\begin{tabular}{|c|c|c|c|c|c|c|c|}
\hline & $\begin{array}{l}\text { e de } \\
\text { de }\end{array}$ & Modelos & & eficientes & $R^{2}$ adj & Sxy & Sxy (\%) \\
\hline & & & $b_{0}$ & $-9,628155$ & & & \\
\hline & & Shumacher & $b_{1}$ & 1,949143 & 0,984 & $\begin{array}{c}0,0138 \\
\left(\mathrm{~m}^{3}\right)\end{array}$ & 7,09 \\
\hline & & & $b_{2}$ & 0,827552 & & & \\
\hline & & & $b_{0}$ & 1,271219 & & & \\
\hline & $\begin{array}{c}a \\
10\end{array}$ & & $b_{1}$ & $-3,904844$ & & & \\
\hline & anos & Schöenfer & $b_{2}$ & 15,747147 & 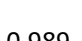 & 0,8664 & 6.97 \\
\hline & & scnoeprer & $b_{3}$ & $-34,598258$ & 0,989 & $(\mathrm{~cm})$ & 0,47 \\
\hline & & & $b_{4}$ & 33,399561 & & & \\
\hline & & & $b_{5}$ & $-11,911940$ & & & \\
\hline & & & $b_{0}$ & $-9,970319$ & & & \\
\hline & & Shumacher & $b_{1}$ & 1,863803 & 0,960 & $\begin{array}{c}0,0422 \\
\left(\mathrm{~m}^{3}\right)\end{array}$ & 6,59 \\
\hline & & & $b_{2}$ & 1,067146 & & & \\
\hline & 11 & & $b_{0}$ & 1,218093 & & & \\
\hline & $\begin{array}{c}A \\
14\end{array}$ & & $b_{1}$ & $-3,892796$ & & & \\
\hline & anos & Schöenfer & $b_{2}$ & 16,383004 & 0989 & 1,2421 & $6 ? 6$ \\
\hline$\underline{\underline{\theta}}$ & & scinvepies & $b_{3}$ & $-35,290522$ & (0,505 & $(\mathrm{cm})$ & 0,20 \\
\hline. & & & $b_{4}$ & 33,048281 & & & \\
\hline ס & & & $b_{5}$ & $-11,467775$ & & & \\
\hline U্ত & & & $b_{0}$ & $-10,282827$ & & & \\
\hline : & & Shumacher & $b_{1}$ & 1,860640 & 0,929 & $\begin{array}{c}0,0698 \\
\left(\mathrm{~m}^{3}\right)\end{array}$ & 7,60 \\
\hline $\bar{\infty}$ & & & $b_{2}$ & 1,181226 & & & \\
\hline & 15 & & $b_{0}$ & 1,202838 & & & \\
\hline & $\begin{array}{c}a \\
18\end{array}$ & & $b_{1}$ & $-3,818645$ & & & \\
\hline & anos & Schöenfer & $b_{2}$ & 16,301312 & 0989 & 1,3513 & 606 \\
\hline & & (3) & $b_{3}$ & $-34,319283$ & כ0כ, & $(\mathrm{cm})$ & \\
\hline & & & $b_{4}$ & 31,072867 & & & \\
\hline & & & $b_{5}$ & $-10,441393$ & & & \\
\hline & & & $b_{0}$ & $-10,093174$ & & & \\
\hline & & Shumacher & $b_{1}$ & 1,919306 & 0,886 & 0,0859 & 9,64 \\
\hline & & & $b_{2}$ & 1,072051 & & & \\
\hline & 19 & & $b_{0}$ & 1,201113 & & & \\
\hline & $\begin{array}{c}a \\
22\end{array}$ & & $b_{1}$ & $-3,892679$ & & & \\
\hline & anos & Schöenfer & $b_{2}$ & 16,808996 & (980 & 1,6162 & 718 \\
\hline & & салертет & $b_{3}$ & $-35,254198$ & 0,000 & $(\mathrm{~cm})$ & 1,40 \\
\hline & & & $b_{4}$ & 31,765588 & & & \\
\hline & & & $b_{5}$ & $-10,630495$ & & & \\
\hline
\end{tabular}
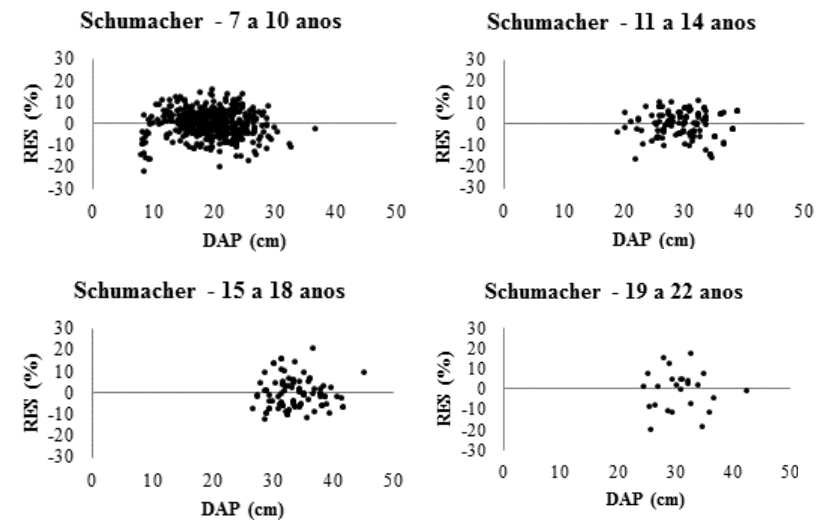

Figura 8. Dispersão dos resíduos do ajuste de volume para o bloco B (Cachoeira). 

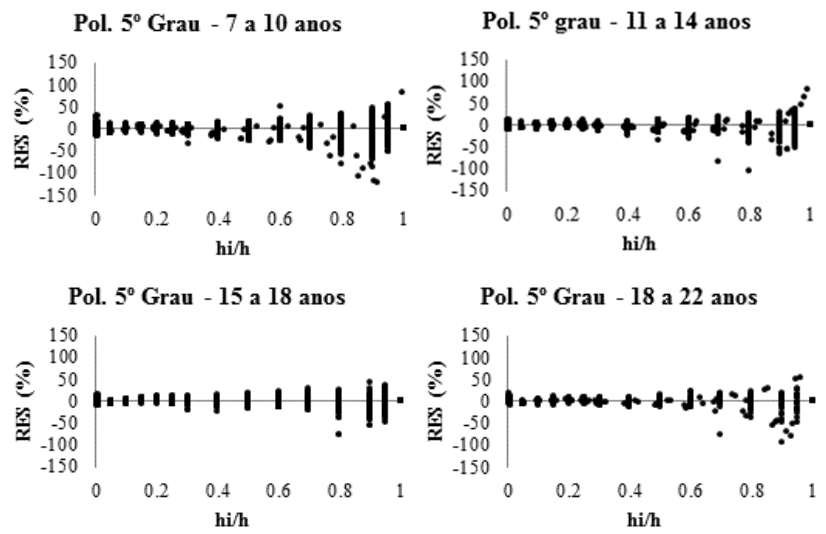

Figura 9. Dispersão dos resíduos do ajuste de afilamento para o bloco B (Cachoeira).

\section{Comparação dos ajustes}

O ajuste por classe de idade mostrou estatísticas semelhantes entre os blocos A e B. Para efeitos de comparação, na Tabela 8 são apresentados valores médios dos erros de estimativa em porcentagem para os modelos de volume de Spurr e de Schumacher-Hall e o modelo de afilamento do Polinômio do 5 o grau em relação às formas de ajuste dos dados, sendo os ajustes feitos: para o conjunto total dos dados, para os blocos (ou regiões A e B) e, posteriormente, em classes de idade para cada bloco.

Observa-se que os valores médios dos erros diminuíram com a estratificação dos dados para o ajuste dos modelos.

Miranda (2012), em uma análise da estratificação dos dados por classe de DAP para modelagem do volume para Eucalyptus sp., encontrou diferença significativa nos resultados, embora o erro padrão de estimativa de ambas as formas de ajuste fora inferior a $10 \%$, além de que quando estimados, os volumes se mostram muito semelhantes independente da forma de ajuste.

Tabela 8. Médias dos erros de estimativa em porcentagem para os modelos de volume e afilamento.

\begin{tabular}{cccc}
\hline \multirow{2}{*}{ Forma de ajuste } & \multicolumn{3}{c}{$\mathbf{S}_{\mathbf{y x}} \%$} \\
\cline { 2 - 4 } & Spurr & Schumacher-Hall & Schöepfer \\
\hline Conjunto de dados & 13,87 & 11,8 & 7,28 \\
\hline Blocos & 10,91 & 10,98 & 7,23 \\
Classe de idade & - & 7,57 & 6,43 \\
\hline
\end{tabular}

\section{CONCLUSÕES}

Todos os modelos ajustados apresentaram estimativas adequadas. Dentre os modelos volumétricos, o de Schumacher-Hall mostrou-se superior ao modelo de Spurr, no ajuste do conjunto total de dados e para os blocos.

Em relação ao modelo de afilamento ajustado, este se mostrou adequado e com estatísticas satisfatórias, de maneira que seus coeficientes podem ser utilizados para determinar volume por sortimento, o que é mais vantajoso para o inventário florestal e planejamento de plantios de P. taeda com a finalidade de obter-se multiprodutos.

A estratificação dos dados por blocos mostrou-se satisfatória, uma vez que os resíduos e erros foram menores. A estratificação por idade revelou-se ainda melhor pelas estatísticas apresentadas e gráficos de resíduos menos tendenciosos em relação ao ajuste apenas por blocos.

\section{AGRADECIMENTOS}

À empresa ADAMI, pela concessão dos dados.

\section{REFERÊNCIAS}

CAMPOS, J.C.C.; LEITE, H.G. Mensuração Florestal: perguntas e respostas. 4.ed. Viçosa: UFV, 2013.

DAVID, H.C. et al. Critérios de estratificação para o ajuste de funções de afilamento em fustes de pinus. Pesquisa Florestal Brasileira, v.34, n.79, p.197-206. 2014.

DRESCHER, R. et al. Fator de forma artificial de Pinus elliotttii Engelm para a região da serra do sudeste do Estado do Rio Grande do Sul. Ciência Rural, v.31, n.1, p.37-42, 2001.

FIGUEIREDO FILHO, A. et al. Compêndio de equações de volume e afilamento de espécies florestais plantadas e nativas para as regiões geográficas do Brasil. Curitiba: os autores, 2014.

IBÁ. Relatório 2017. 2017. Disponível em: http://iba.org/images/shared/Biblioteca/IBA_RelatorioAnual201 $6 \_. p d f$

KOHLER, S.V. et al. Diferentes métodos de ajuste do modelo volumétrico de Schumacher e Hall. In: Congresso Florestal Paranaense, 2012, Curitiba. Anais... Curitiba: UNICENTRO, 2012.

KOHLER, S.V. et al. Modelos de afilamento para Pinus taeda por classe de idade. Floresta e Ambiente, v.20, n.4, p.470-479, 2013.

LEITE, H.G.; ANDRADE, V.C.L. Importância das variáveis altura dominante e altura total em equações hipsométricas e volumétricas. Árvore, v.27, n.3, p.301-310, 2003.

MACHADO, S.A. et al. Comparação de modelos de afilamento do tronco para diferentes idades e regimes de desbastes em plantações de Pinus oocarpa Schiede. Boletim de Pesquisa Florestal, n.48, p.41-64, 2004.

MIRANDA, R.O.V. Modelagem em nível de povoamento para Eucalyptus sp. com estratificação em classes de precipitação pluviométrica. 2012. 78p. (Dissertação de mestrado). 
OLIVEIRA, M.L.R. et al. Estimação do volume de árvores de clones de eucalipto pelo método da similaridade de perfis. Revista Árvore, v.33, n.1, p.133-141, 2009.

OPTIMBER. Guia de instruções do usuário: Florexcel. OpTimber, 2016.

PRODAN, M. et al. Mensura forestal. San José: GTZ-IICA, 1997.

SCOLFORO, J.R. Mensuração Florestal 3: relações quantitativas em volume, peso e a relação hipsométrica. Lavras, MG: ESAL/FAEPE, 1993.

SOUZA, C.A.M. Modelos de afilamento para Pinus taeda L. ajustados segundo a forma do tronco e métodos de estratificação. 2009. 120p. (Tese de doutorado).

YOSHITANI JUNIOR, M. Avaliação biométrica e econômica de plantios de Pinus taeda L. em Santa Catarina. 2009. 115p. (Dissertação de mestrado). 